\title{
A new extremely low-mass white dwarf in the NLTT catalogue ${ }^{\star}$
}

\author{
A. Kawka and S. Vennes
}

\begin{abstract}
Astronomický ústav, Akademie věd České republiky, Fričova 298, 25165 Ondřejov, Czech Republic e-mail: [kawka; vennes] @sunstel.asu.cas.cz
\end{abstract}

Received 22 July 2009 / Accepted 14 September 2009

\begin{abstract}
We report on the discovery of the extremely low-mass, hydrogen-rich white dwarf, NLTT 11748. Based on measurements of the effective temperature $(8540 \pm 50 \mathrm{~K})$ and surface gravity $(\log g=6.20 \pm 0.15)$ obtained by fitting the observed Balmer line profiles with synthetic spectra, we derive a mass of $0.167 \pm 0.005 M_{\odot}$. This object is one of only a handful of white dwarfs with masses below $0.2 M_{\odot}$ that are believed to be the product of close binary evolution with an episode of Roche lobe overflow onto a degenerate companion (neutron star or white dwarf). Assuming membership in the halo population, as suggested by the kinematics and adopting a cooling age of 4.0-6.3 Gyr for the white dwarf, we infer a progenitor mass of 0.87-0.93 $M_{\odot}$. The likely companion has yet to be identified, but a search for radial velocity variations may help constrain its nature.
\end{abstract}

Key words. white dwarfs - stars: individual: NLTT 11748 - stars: atmospheres

\section{Introduction}

The average mass of white dwarfs is approximately $0.6 M_{\odot}$, with the bulk of these stars having a mass near this average value. Additional smaller peaks at the ultramassive and low-mass ends of the mass distribution are observed. In the case of low-mass white dwarfs $\left(M<0.4 M_{\odot}\right)$, the galaxy is not old enough to have produced such objects. Several low-mass white dwarfs $\left(M<0.45 M_{\odot}\right)$ have been checked for radial velocity variations (e.g., Maxted et al. 2000; Nelemans et al. 2005); however, not all low-mass white dwarfs were found to be variable. A statistical analysis of the incidence of binarity among low-mass white dwarfs was conducted by Napiwotzki et al. (2007) using the ESO SN Ia progenitor survey, which included 26 white dwarfs with masses lower than $0.42 M_{\odot}$. From this sample they find only 11 with variable radial velocities showing that single low-mass white dwarfs do exist. It may be that some of these low-mass white dwarfs have indeed formed through single-star evolution. Kilic et al. (2007c) argues that up to 50\% of low-mass helium core white dwarfs $M \sim 0.4 M_{\odot}$ could have formed from progenitors with supersolar metallicities.

Although some low-mass white dwarfs may evolve through single-star evolution, no single-star evolutionary path is known for extremely low-mass (ELM) white dwarfs $\left(M<0.2 M_{\odot}\right)$, which were initially identified as companions to pulsars (e.g., van Kerkwijk et al. 1996; Bassa et al. 2006). Subsequently, several low-mass white dwarfs have been discovered in the Sloan Digitial Sky Survey (Kleinman et al. 2004; Eisenstein et al. 2006), with SDSS J123410.37-022802.9 having the lowest mass at $\sim 0.18 M_{\odot}$ (Liebert et al. 2004). Other spectroscopic identifications include the high-velocity white dwarf LP400-22, with a mass of $0.17 \pm 0.01 M_{\odot}$ (Kawka et al. 2006), and

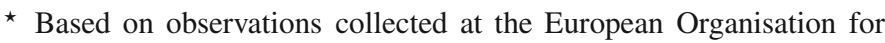
Astronomical Research in the Southern Hemisphere, Chile under programme ID 82.D-0750.
SDSS J0917+46, which was discovered as part of a search for hypervelocity B-type stars (Kilic et al. 2007a). Follow-up observations of the last two of these stars using the $6.5 \mathrm{~m}$ MMT telescope found them to have variable radial velocities. SDSS J0917+46 was found to be in a binary with an orbital period of $7.5936 \pm 0.0024 \mathrm{~h}$ (Kilic et al. 2007b) and LP 400-22 was found to have an orbital period of approximately 1 day (Kilic et al. 2009; Vennes et al. 2009). In both cases, the companion remains unknown, but a white dwarf, neutron star, or a low-mass main-sequence star were considered as possible companions.

The recent discovery of ELM white dwarfs offers new challenges for binary evolution scenarios, as well as for calculating the internal structure of white dwarfs including the effect of residual burning and model atmospheres in a poorly explored range of stellar parameters.

We present our discovery and analysis of the ELM white dwarf NLTT 11748. Our spectroscopic and photometric observations are presented in Sect. 2. We present our model atmosphere analysis of the object in Sect. 3 and discuss our results in Sect. 4. We summarise in Sect. 5.

\section{Observations}

We obtained low-resolution spectroscopy of NLTT 11748 using the EFOSC2 (ESO Faint Object Spectrograph and Camera) attached to the New Technology Telescope (NTT) at La Silla Observatory. The 300 lines per $\mathrm{mm}$ grating with a blaze wavelength of $4000 \AA$ (Grism 11) was used to obtain two spectra with a resolution of $16 \AA$. The exposure time for each spectrum was $20 \mathrm{~min}$. The slitwidth was set to 1 arcsec. We acquired both spectra on UT 2008 October 21 in photometric conditions with the seeing varying between 1 and 1.3 arcsec throughout the night. A wavelength calibration spectrum of helium and argon immediately followed the NLTT11748 spectra. The spectra were 
Table 1. Photometry of NLTT 11748.

\begin{tabular}{lcc}
\hline \hline Band & Measurement & Model $^{a}$ \\
\hline FUV & $21.644 \pm 0.291$ & 21.839 \\
NUV & $18.673 \pm 0.047$ & 18.494 \\
$V$ & $16.5 \pm 0.3$ & 16.493 \\
$J$ & $15.873 \pm 0.077$ & 15.933 \\
$H$ & $15.777 \pm 0.137$ & 15.768 \\
$K_{\mathrm{S}}$ & $16.904:$ & 15.700 \\
\hline
\end{tabular}

${ }^{a}$ Based on best model fit including $\left(E(B-V), R_{V}\right)=(0.10,3.2)$. See Sect. 4.

flux-calibrated using the flux standard Feige 110, which was obtained on the same night.

We then obtained ultraviolet photometry from the Galaxy Evolution Explorer (GALEX) all sky survey. GALEX provides photometry in two bands, FUV and NUV. The FUV bandpass is 1344-1786 ̊ with an effective wavelength of $1528 \AA$, and the NUV bandpass is 1771-2831 $\AA$ with an effective wavelength of $2271 \AA$. We also obtained infrared photometry from the Two Micron All Sky Survey (2MASS), which is available at VizieR at the Centre de Données astronomique $(\mathrm{CDS})^{1}$. Table 1 summarises the available photometry. The $V$ band photometry is estimated from our flux calibrated spectroscopy. We estimated the uncertainty in the $V$ band to be $0.3 \mathrm{mag}$, which includes systematic effects of varying seeing and data reduction procedures. The $2 M A S S K_{\mathrm{S}}$ band is uncertain and is not considered further.

\section{Analysis}

We first fitted the observed Balmer lines with a grid of pure hydrogen synthetic spectra that extends from 5000 to $7000 \mathrm{~K}$ (in steps of $500 \mathrm{~K}$ ), 8000 to $16000 \mathrm{~K}$ (in steps of $1000 \mathrm{~K}$ ), 18000 to $32000 \mathrm{~K}$ (in steps of $2000 \mathrm{~K}$ ), and 36000 to $100000 \mathrm{~K}$ (in steps of $4000 \mathrm{~K}$ ) at $\log g=6.0$ to 9.5 (in steps of $0.25 \mathrm{dex}$ ). Following initial parameter estimations for NLTT 11748, we calculated a denser grid with temperatures ranging from $8000 \mathrm{~K}$ to $9000 \mathrm{~K}$ (in steps of $100 \mathrm{~K}$ ) and surface gravities ranging from $\log g=6.0$ to 7.0 (in steps of $0.25 \mathrm{dex}$ ). Figure 1 shows the observed Balmer line profiles compared to the best-fit model spectrum. This model spectrum corresponds to an effective temperature of $T_{\text {eff }}=8540 \pm 50 \mathrm{~K}$ and a surface gravity of $\log g=6.20 \pm$ 0.15 . The quoted uncertainties are only statistical $(1 \sigma)$. In general surface gravity measurements may be effected by a systematic offset between the assumed and the actual spectral resolution. At low surface gravity, a $1 \AA$ offset would correspond to $\approx 0.08$ dex shift in surface gravity or $0.007 M_{\odot}$.

Using the evolutionary mass-radius relations of Serenelli et al. (2002), we obtain a mass of $0.167 \pm 0.005 M_{\odot}$ and a cooling age of 4.0-6.3 Gyr. The use of low-metallicity models from Serenelli et al. (2002) is justified by the old age of the star. We also calculated the absolute magnitude $\left(M_{V}\right)$ using these models. For comparison we also calculated a mass of $0.164 \pm 0.005 M_{\odot}$ using the solar metallicity models of Serenelli et al. (2001), which is very similar to the low-metallicity mass determination. However, the cooling age of the system assuming solar metallicity would be between 1 and $4 \mathrm{Gyr}$, which is significantly lower than the cooling age predicted by the lowmetallicity models. The difference in the cooling age can be attributed to the effect of metallicity on the residual hydrogen

${ }^{1}$ http://cdsweb.u-strasbg.fr/CDS.html

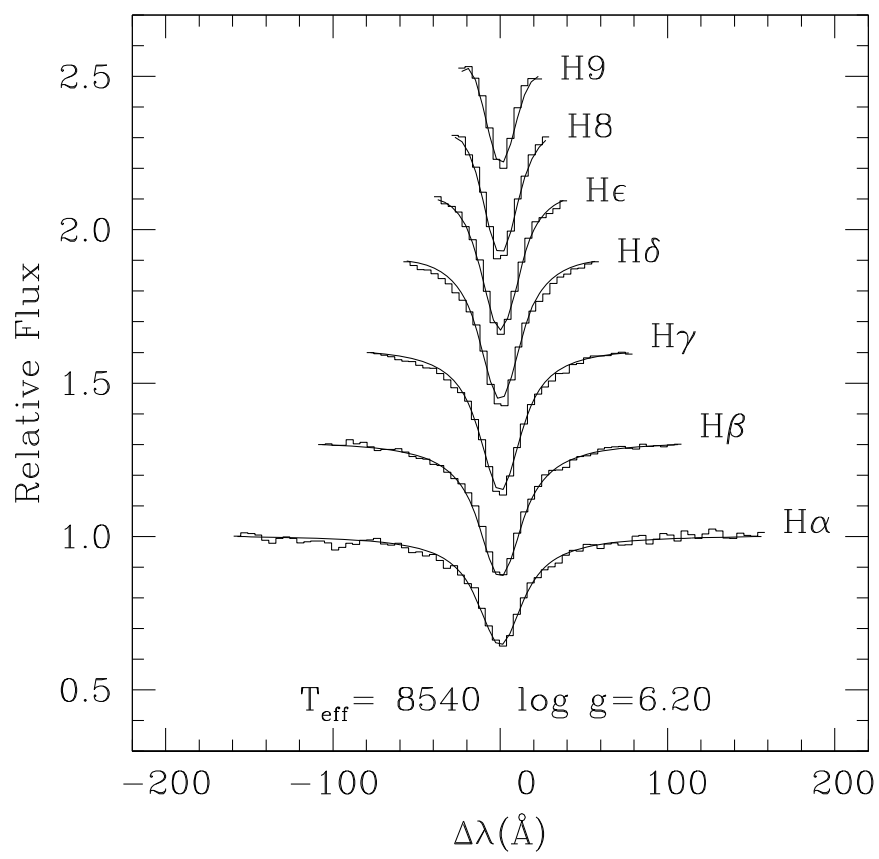

Fig. 1. Balmer line profiles of NLTT 11748 compared to the best-fit model spectrum with an effective temperature of $T_{\text {eff }}=8540 \pm 50 \mathrm{~K}$ and a surface gravity of $\log g=6.20 \pm 0.15$.

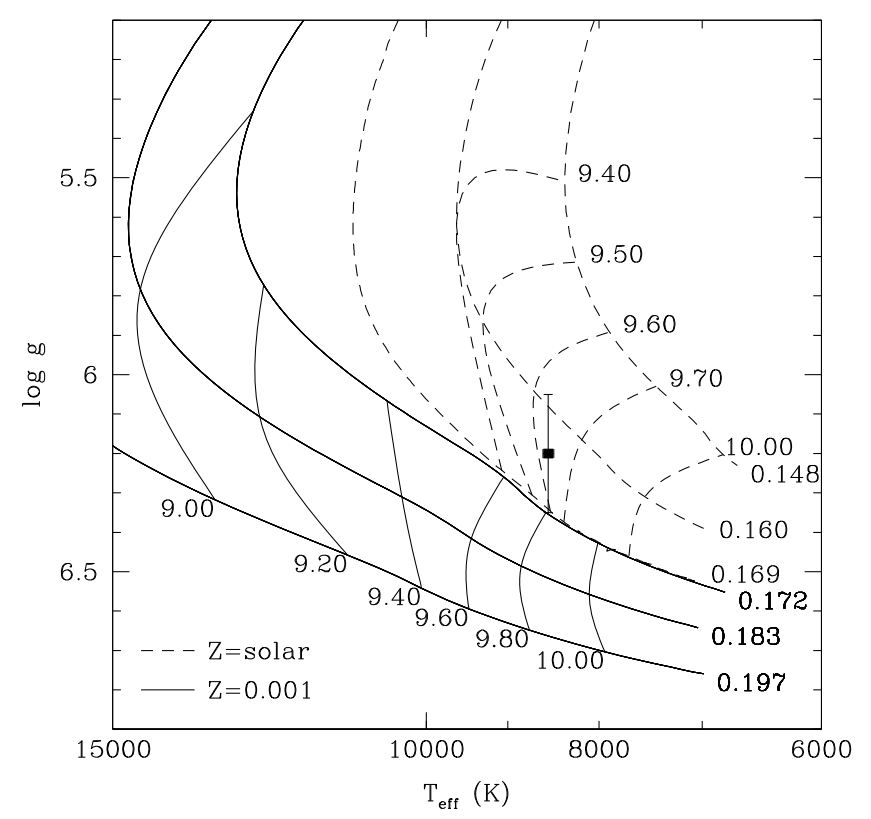

Fig. 2. The effective temperature and surface gravity of NLTT 11748 compared to evolutionary mass-radius relations. The models with solar metallicity and low metallicity $(Z=0.001)$ are from Serenelli et al. (2001) and Serenelli et al. (2002), respectively. The isochrones are labelled with $\log$ (years) and the curves of constant mass are in units of $M_{\odot}$.

burning, which is expected for white dwarfs with such a low mass with solar or low-metallicity progenitors (Serenelli et al. 2002). Figure 2 shows the effective temperature and surface gravity of NLTT 11748 compared to the evolutionary massradius relations of Serenelli et al. (2001) and Serenelli et al. (2002). Table 2 lists our adopted values for $T_{\mathrm{eff}}, \log g, M$, and $M_{V}$. 
Table 2. Properties of NLTT 11748.

\begin{tabular}{llc}
\hline \hline Parameters & Measurement & Reference \\
\hline RA (J2000.0) & 034516.83 & 1 \\
Dec (J2000.0) & +174808.71 & 1 \\
Effective temperature & $8540 \pm 50 \mathrm{~K}$ & 2 \\
Surface gravity & $6.20 \pm 0.15$ & 2 \\
Mass & $0.167 \pm 0.005 M_{\odot}$ & 2 \\
$M_{V}$ & $9.72 \pm 0.33$ & 2 \\
Distance & $199 \pm 40 \mathrm{pc}$ & 2 \\
$v_{\text {rad }}$ & $415 \pm 50 \mathrm{~km} \mathrm{~s}^{-1}$ & 2 \\
Proper motion & $\mu_{\mathrm{RA}}=0.2361 \pm 0.0043^{\prime \prime} \mathrm{yr}^{-1}$ & 1,3 \\
& $\mu_{\text {Dec }}=-0.1792 \pm 0.0043^{\prime \prime} \mathrm{yr}^{-1}$ & 1,3 \\
Kinematics & $U=-406 \pm 57 \mathrm{~km} \mathrm{~s}^{-1}$ & 2 \\
& $V=-219 \pm 66 \mathrm{~km} \mathrm{~s}^{-1}$ & 2 \\
& $W=-161 \pm 35 \mathrm{~km} \mathrm{~s}^{-1}$ & 2 \\
\hline
\end{tabular}

References: (1) Salim \& Gould (2003); (2) this work; (3) Lépine \& Shara (2005).

\section{Discussion}

\subsection{Kinematics}

We selected NLTT 11748 (LP413-40) as a white dwarf candidate from the revised NLTT catalogue (Salim \& Gould 2003) using the reduced proper motion diagram (Salim \& Gould 2002; Kawka et al. 2004). Luyten (1977) originally listed NLTT 11748 in his white dwarf catalogue, and, more recently the star has also been recovered in the LSPM catalogue as LSPM J0345+1748 (Lépine \& Shara 2005). The rNLTT and LSPM catalogue remeasured the proper motions independently. We list the weighted average of these measurements in Table 2. We also measured the radial velocity of the star using the Balmer line series $(\mathrm{H} \alpha$ to $\mathrm{H} \gamma$ ) and the corresponding heliocentric velocity is provided in Table 2.

The photometric distance estimate toward NLTT 11748 is possibly effected by extinction in the line of sight. NLTT 11748 is near the Taurus-Auriga molecular cloud, which is a well known star formation region located at a distance of $\sim 140 \mathrm{pc}$ (e.g., Li \& Hu 1998). The extinction in the line of sight toward NLTT 11748 based on the maps of Schlegel et al. (1998) is $E(B-V)=0.2828$, which corresponds to a large extinction in the ultraviolet. Assuming $R_{V}=3.2$ and using the relations from Cardelli et al. (1989), we estimate the extinction in the NUV and FUV bands to be $A_{\mathrm{NUV}}=2.6$ and $A_{\mathrm{FUV}}=2.3$, respectively. However, we do not expect such a large extinction because the object is still well within the plane.

The extinction in the infrared should be very small, so we calculated a distance of $199 \pm 40$ pc using the $J$ magnitude. Using the effective temperature and surface gravity from the spectral fit, we calculated the absolute magnitude in the FUV, NUV, $V, J$, and $H$ bands. The expected NUV $-J$ for the white dwarf is 1.90 , but the observed NUV $-J$ colour is $2.836 \pm$ 0.090 . This corresponds to an extinction of $A_{\mathrm{NUV}}=0.935$ in the NUV and $E(B-V) \approx 0.10$. Using the synthetic spectrum of the white dwarf at the derived temperature and surface gravity, we calculated a synthetic spectrum taking extinction into account where $E(B-V)=0.10$ and $R_{V}=3.2$. Figure 3 shows the observed energy distribution compared to a synthetic spectrum with and without extinction. The corresponding model magnitudes including extinction are given in Table 1.

Based on the proper motion, distance, and velocity measurements, we calculated the kinematics (Table 2) following Johnson \& Soderblom (1987) and assuming that the solar motion

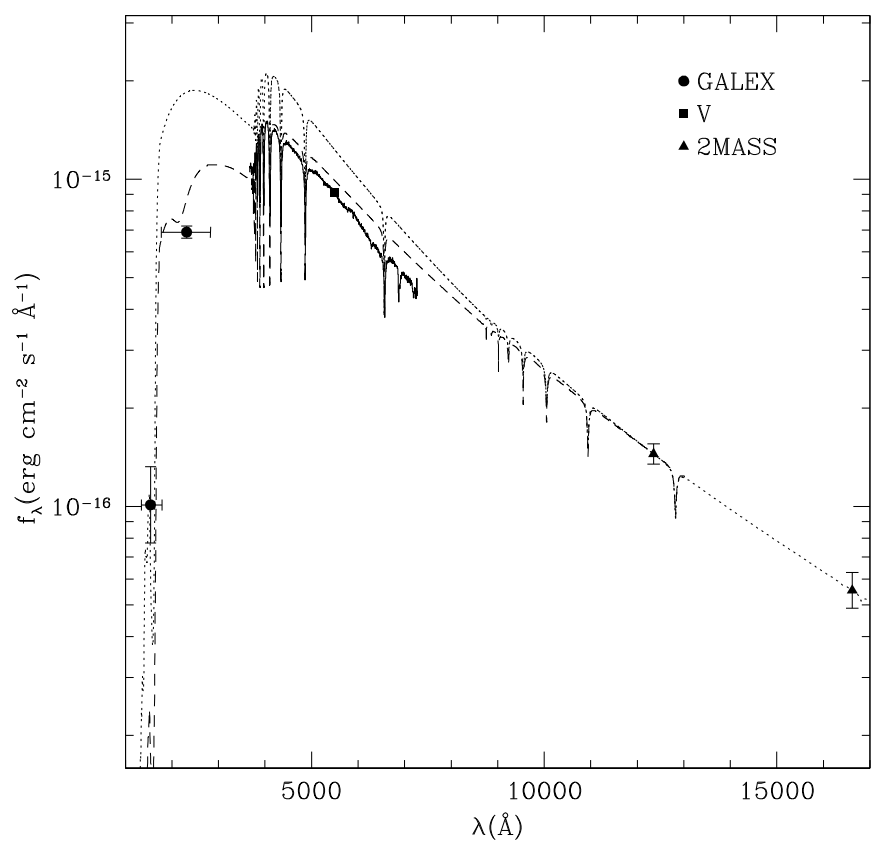

Fig. 3. Energy distribution of NLTT 11748 combining all available data compared to our hydrogen-rich spectrum at $T_{\text {eff }}=8500 \mathrm{~K}$ and $\log g=6.25$ without extinction (dotted line) and with extinction assuming $E(B-V)=0.10$ (dashed line). The filled circles are the observed data, and the empty circles the predicted photometric points if we assume no extinction.

relative to the local standard of rest (LSR) is $\left(U_{\odot}, V_{\odot}, W_{\odot}\right)=$ $(10.1,4.0,6.7)$ as determined by Hogg et al. (2005). Figure 4 shows the $U$ and $V$ velocities of NLTT 11748 assuming a radial velocity of $415 \mathrm{~km} \mathrm{~s}^{-1}$. However, NLTT 11748 probably resides in a close binary, and the correct systemic velocity still needs to be measured ${ }^{2}$. We illustrate the possible variations in $U V$ velocities assuming a range of values from 0 to $600 \mathrm{~km} \mathrm{~s}^{-1}$ for the systemic velocity. NLTT 11748 bears a striking resemblance with the other high-velocity ELM white dwarf LP 400-22 (Kilic et al. 2009; Vennes et al. 2009).

\subsection{Binary evolution}

Given that the two field ELM white dwarfs (LP 400-22, SDSS $\mathrm{J} 0917+46)$ that were checked for radial velocity variations were found to be in a binary system, it is highly probable that NLTT 11748 is also in a binary system. In the two confirmed field systems, the companion remains unknown, although the other known ELM white dwarfs are companions to pulsars (van Kerkwijk et al. 1996; Bassa et al. 2006), so it is possible that NLTT 11748 has a neutron star companion. If we assume that the companion is a neutron star, then the orbital period is expected to be $\sim 10-15 \mathrm{~h}$ based on the mass versus orbital period relations for He WDs with a neutron star companion (Benvenuto $\&$ De Vito 2005). We searched for radio sources in the vicinity of NLTT 11748 using VizieR, and the nearest source is an unrelated object 2.5 arcmin away.

It is also possible that the companion is another, more massive white dwarf. Nelemans et al. (2001) present a population synthesis of double degenerate systems that experience at least two phases of mass transfer, out of which at least one will result

\footnotetext{
2 The measured velocity difference between the two available spectra is $\sim 40 \mathrm{~km} \mathrm{~s}^{-1}$, which suggests the star is showing velocity variations, but the $1 \sigma$ uncertainties of the two measurements overlap.
} 


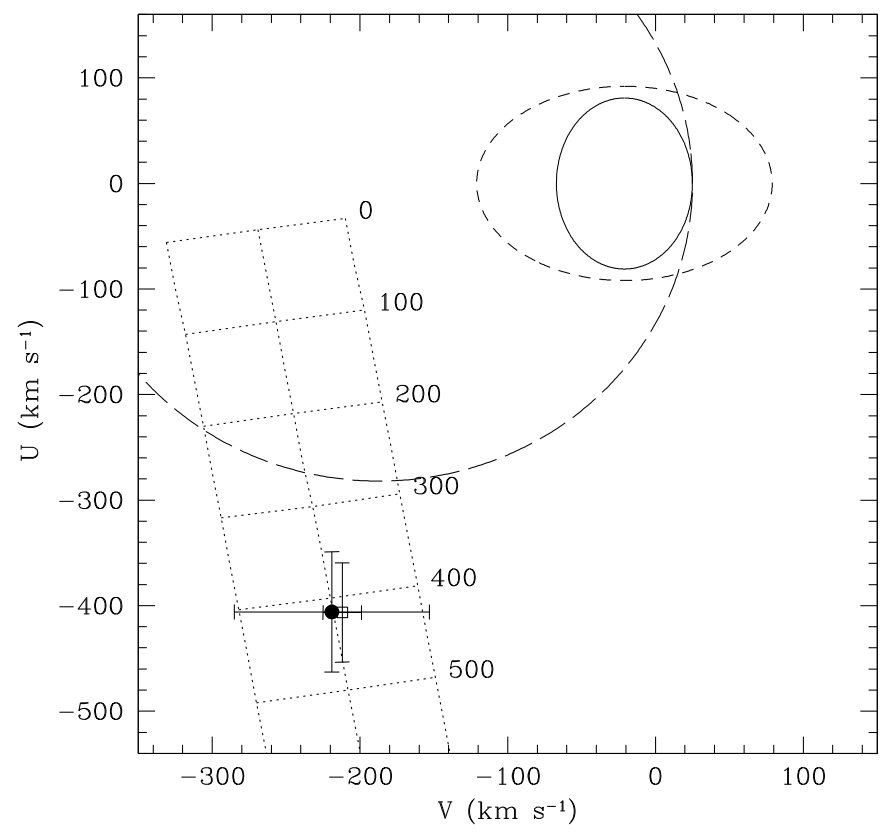

Fig. 4. The $U$ and $V$ velocities of NLTT 11748 (full circle) and LP 400-22 (open square) compared to the $2 \sigma$ thin- (full line) and thick(short dashed line) disk ellipses and the $2 \sigma$ halo ellipse (long dashed line). The predicted $U V$ velocities (dotted line) for varying radial velocity measurements are shown with the assumed velocity.

in a common envelope allowing the white dwarfs to spiral in. Their population synthesis also shows a correlation between the mass of the brighter white dwarf and the orbital period.

Although unlikely, the companion could be a low-mass main sequence star. Our optical spectrum and 2MASS photometry do not show any evidence of a cool companion. Since NLTT 11748 is relatively faint, 2MASS provides a reliable measurement in the $J$ band, with $H$ having a considerably greater uncertainty. The absolute $J$ magnitude for the white dwarf is $M_{J}=9.3$. A red dwarf of spectral type M5 to M5.5 would have equally contributed in this spectral region (Kirkpatrick \& McCarthy 1994), so a prospective late type companion would have to be much less massive than $0.2 M_{\odot}$.

Evolutionary scenarios that produce ELM white dwarfs involve an episode of Roche lobe overflow and accretion onto a degenerate companion that stripped the envelope of the white dwarf progenitor leaving a low-mass degenerate core (Willems \& Kolb 2002; Benvenuto \& De Vito 2005). Assuming halo membership, as suggested by the kinematics, the progenitor lifetime on the main-sequence would range from 7 to 9 Gyr corresponding to a late $\mathrm{G}$ star with a mass of $0.87-0.93 M_{\odot}$ (Girardi et al. 2000).

\section{Conclusions}

Based on a model atmosphere analysis, we have shown that NLTT 11748 is an ELM white dwarf $\left(M=0.167 \pm 0.005 M_{\odot}\right)$. Although we assumed a pure hydrogen composition, near ultraviolet spectroscopy is required to confirm our model atmosphere analysis and to constrain the abundance of heavy elements in the atmosphere. NLTT 11748 is one of only a handful of low-gravity white dwarfs that allow a test of our models in these unique conditions.

The star almost certainly belongs to a close binary system with a still unidentified degenerate companion. A probable evolutionary scenario involves a post supernova system comprising an evolving $G$ star and a neutron star that experienced Roche lobe overflow accretion, leaving an ELM degenerate star. This object would be part of a growing family of low-mass Xray binary remnants. A radial velocity study should be able to constrain the orbital period and the mass function of the unseen companion. On the other hand, the binary companion may be another white dwarf, thereby confirming another formation channel for ELM white dwarfs in parallel with the channel involving a neutron star. Our kinematic study shows that the system belongs to the Galactic halo, which helps constrain the age, hence the mass of the donor star.

Finally, studies of ELM white dwarfs are useful in exploring the effect of residual burning on white dwarf mass-radius relations. In this regard an independent estimate of the radius using parallax measurements is essential. In addition, as more ELM white dwarfs are discovered, a more comprehensive set of evolutionary tracks for these stars is required.

Acknowledgements. A.K. and S.V. are supported by grants IAA301630901 and IAA300030908 from the GA AV, respectively. A.K. also acknowledges support from the Centre for Theoretical Astrophysics (LC06014). This research has made use of the VizieR catalogue access tool, CDS, Strasbourg, France. This publication makes use of data products from the 2MASS, which is a joint project of the University of Massachusetts and the Infrared Processing and Analysis Center/California Institute of Technology, funded by the National Aeronautics and Space Administration and the National Science Foundation.

\section{References}

Bassa, C. G., van Kerkwijk, M. H., Koester, D., \& Verbunt, F. 2006, A\&A, 456, 295

Benvenuto, O. G., \& De Vito, M. A. 2005, MNRAS, 362, 891

Cardelli, J. A., Clayton, G. C., \& Mathis, J. S. 1989, ApJ, 345, 245

Eisenstein, D. J., Liebert, J., Harris, H. C., et al. 2006, ApJS, 167, 40

Girardi, L., Bressan, A., Bertelli, G., \& Chiosi, C. 2000, A\&AS, 141, 371

Hogg, D. W., Blanton, M. R., Roweis, S. T., \& Johnston, K. V. 2005, ApJ, 629, 268

Johnson, D. R. H., \& Soderblom, D. R. 1987, AJ, 93, 864

Kawka, A., Vennes, S., \& Thorstensen, J. R. 2004, AJ, 127, 1702

Kawka, A., Vennes, S., Oswalt, T. D., Smith, J. A., \& Silvestri, N. M. 2006, ApJ, 643, L123

Kilic, M., Allende Prietro, C., Brown, W. R., \& Koester, D. 2007a, ApJ, 660, 1451

Kilic, M., Brown, W. R., Allende Prietro, C., Pinsonneault, M. H., \& Kenyon, S. J. 2007b, ApJ, 664, 1088

Kilic, M., Stanek, K. Z., \& Pinsonneault, M. H. 2007c, ApJ, 671, 761

Kilic, M., Brown, W. R., Allende Prietro, C., et al. 2009, ApJ, 695, L92

Kirkpatrick, J. D., \& McCarthy, Jr., D. W. 1994, AJ, 107, 333

Kleinman, S. J., Harris, H. C., Eisenstein, D. J., et al. 2004, ApJ, 607, 426

Lépine, S., \& Shara, M. M. 2005, AJ, 129, 1483

Liebert, J., Bergeron, P., Eisenstein, D., et al. 2004, ApJ, 606, L147

Li, J. Z., \& Hu, J. Y. 1998, A\&AS, 132, 173

Luyten, W. J. 1977, White Dwarfs II, Univ. of Minnesota, Minneapolis

Maxted, P. F. L., Marsh, T. R., \& Moran, C. K. J. 2000, MNRAS, 319, 305

Napiwotzki, R., Karl, C. A., Nelemans, G., et al. 2007, in 15th European

Workshop on White Dwarfs, ed. R. Napiwotzki, \& M. R. Burleigh (San Francisco: ASP), ASP Conf. Ser., 372, 387

Nelemans, G. Yungelson, L. R., Portegies Zwart, S. F., \& Verbunt, F. 2001, A\&A, 365,491

Nelemans, G., Napiwotzki, R., Karl, C., et al. 2005, A\&A, 440, 1087

Salim, S., \& Gould, A. 2002, ApJ, 575, L83

Salim, S., \& Gould, A. 2003, ApJ, 582, 1011

Schlegel, D. J., Finkbeiner, D. P., \& Davis, M. 1998, ApJ, 500, 525

Serenelli, A. M., Althaus, L. G., Rohrmann, R. D., \& Benvenuto, O. G. 2001, MNRAS, 325, 607

Serenelli, A. M., Althaus, L. G., Rohrmann, R. D., \& Benvenuto, O. G. 2002, MNRAS, 337, 1091

van Kerkwijk, M. H., Bergeron, P., \& Kulkarni, S. R. 1996, ApJ, 467, L89

Vennes, S., Kawka, A., Vaccaro, T. R., \& Silvestri, N. M. 2009, A\&A, submitted

Willems, B., \& Kolb, U. 2002, MNRAS, 337, 1004 